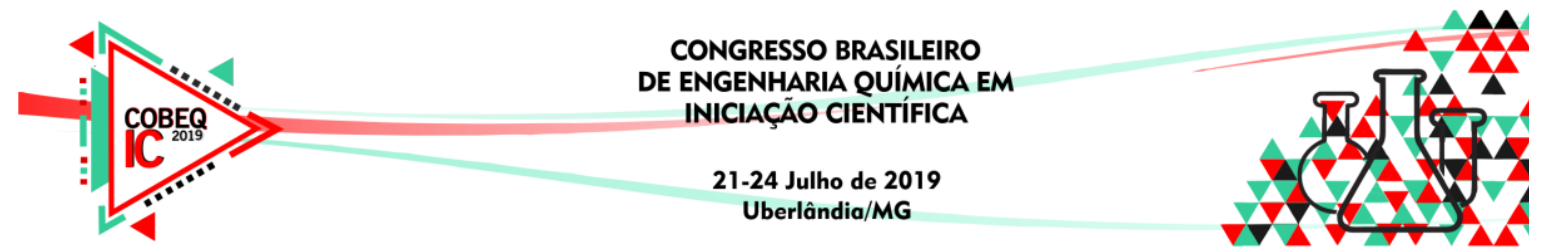

\title{
SÍNTESE DE MICROPARTÍCULAS POLIMÉRICAS POROSAS COM CARACTERÍSTICAS ADSORVENTES APLICADAS NO TRATAMENTO DE ÁGUA
}

\author{
C. O. MEDEIROS ${ }^{1 *}$, M. L. GASPAROTTE ${ }^{1 *}$, R. D. SEIXAS $^{1 *}$, M. G. F. TORRAGA ${ }^{1}$ e R. \\ GIUDICI $^{1}$
}

${ }^{1}$ Universidade de São Paulo - Escola Politécnica, Departamento de Engenharia Química E-mail para contato: camila.medeiros@usp.br, maiara.gasparotte@usp.br, raquel.seixas@usp.br, maria.giuliana@usp.br, rgiudici@usp.br

\begin{abstract}
RESUMO - A síntese de micropartículas poliméricas porosas para aplicação no tratamento de água contaminada com corantes foi estudada dos pontos de vista de cinética das reações e potencial de adsorção. O núcleo de poli (estireno-co-anidrido maleico) foi sintetizado por copolimerização alternada de anidrido maleico e estireno via polimerização por dispersão e, seu invólucro, por precipitação, com os agentes reticulante (divinilbenzeno) e porogênico (n-heptano). Para a funcionalização destas partículas com grupos carboxilato, foi utilizado hidróxido de sódio. A conversão dos monômeros foi medida por gravimetria e a caracterização de diâmetro e superfície das partículas foi realizada por espalhamento dinâmico de luz e microscopia eletrônica de varredura, respectivamente. Por fim, mediram-se com UV/Vis as absorbâncias de soluções de azul de metileno antes e depois dos testes de adsorção. Obteve-se moderada conversão de monômeros em partículas esféricas e estáveis, que apresentaram alta capacidade adsortiva de corante (capacidade máxima de 1240,69 mg/g), características que demonstram a viabilidade do método utilizado.
\end{abstract}

\section{INTRODUÇÃO}

Efluentes industriais podem possuir corantes, cujo uso aumentou ao longo dos anos, sendo muito utilizados em indústrias têxteis, farmacêuticas, alimentícias, de cosméticos, papel e celulose, tintas e impressão. A descarga é altamente colorida, uma vez que enorme quantidade de corantes permanece inalterada durante a coloração e a lavagem (Santos e Boaventura, 2008). Assim, o tratamento deste efluente é crucial para a preservação do meio ambiente.

A remediação de poluentes via processos de adsorção é considerada uma das técnicas mais promissoras devido à simplicidade de operação, alta eficiência e aplicabilidade no tratamento de efluentes em larga escala. Para atender a esta demanda, grandes esforços têm sido dedicados à síntese de materiais adsorventes avançados (Chen et al., 2016; Qin et al., 2016). Em paralelo, devido ao crescimento no campo de micromateriais nas últimas duas décadas, tem-se testemunhado o rápido desenvolvimento de adsorventes microestruturados de alto desempenho.

*As autoras contribuíram igualmente para a elaboração do manuscrito. 


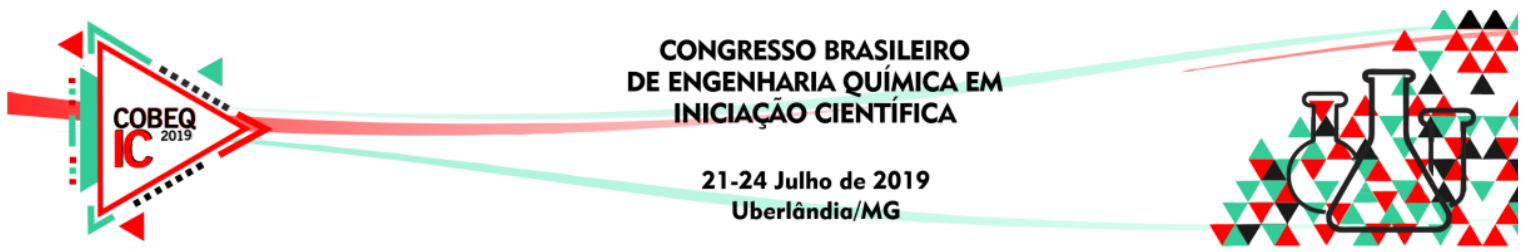

Vários polímeros com porosidades definidas e propriedades superficiais controláveis foram sintetizados com este fim, tendo em vista a necessidade de uma extensa superfície de contato com sítios de ligação para adsorver poluentes. No presente estudo, realizou-se a polimerização, purificação e funcionalização de partículas esféricas, estáveis, altamente porosas e reticuladas, com base no trabalho de Chen et al. (2016).

\section{METODOLOGIA EXPERIMENTAL}

A síntese das micropartículas poliméricas ocorreu em um reator encamisado agitado constantemente e foi dividida em três etapas: 1) síntese do núcleo, 2) síntese da casca porosa e 3) remoção do núcleo. A primeira parte consistiu na polimerização por dispersão entre anidrido maleico (AM) e estireno, com iniciador azobisisobutironitrila (AIBN) no solvente acetato de isopentila, sem agitação. Na segunda parte, adicionaram-se divinilbenzeno (DVB, agente reticulante) e n-heptano (agente porogênico) em uma reação de polimerização por precipitação, com agitação. A terceira etapa envolveu a lavagem das suspensões com acetona, centrifugação e secagem a vácuo, seguida pela funcionalização das partículas com solução de hidróxido de sódio, conforme a Figura 1.

Figura 1 - Etapas da síntese das partículas ocas e porosas.

\begin{tabular}{|c|c|c|}
\hline Etapa 1 & Etapa 2 & Etapa 3 \\
& & \\
Síntese do núcleo & Síntese da casca porosa & Remoção do núcleo \\
\hline
\end{tabular}

A conversão do processo foi determinada por análise gravimétrica. As amostras receberam acetona em cápsulas de alumínio e foram mantidas em estufa até a completa evaporação dos componentes voláteis, quando mediu-se a massa final de polímero seco. Os diâmetros foram determinados com o Zetasizer Nano ZS90 da Malvern, o qual se baseia no espalhamento dinâmico de luz (EDL). A fim de avaliar a aparência tridimensional das partículas, obtiveram-se imagens no microscópio eletrônico de varredura (MEV). A funcionalização foi feita com a hidrólise dos grupos anidrido e formação de carboxilatos na casca, adicionando-se $30 \mathrm{~mL}$ de solução de hidróxido de sódio $\left(\mathrm{NaOH}, 1 \mathrm{molL}^{-1}\right)$ a $2 \mathrm{~g}$ de micropartículas. A reação ocorreu durante $5 \mathrm{~h}$ sob agitação magnética, à temperatura ambiente.

Para a construção da curva de calibração do azul de metileno, foram preparadas soluções padrão de concentrações conhecidas. Os pHs foram determinados com pHmetro e, as absorbâncias, com espectrômetro UV/Vis no comprimento de onda de $633 \mathrm{~nm}$. Nos ensaios de adsorção, as micropartículas funcionalizadas foram dispersas em solução aquosa de corante em concentrações variando de $0 \mathrm{mmolL}^{-1}$ a $10 \mathrm{mmolL}^{-1}$, a $25^{\circ} \mathrm{C}$, pH 7 e durante $24 \mathrm{~h}$ sob agitação magnética. Finalmente, as soluções foram centrifugadas e o líquido sobrenadante teve sua absorbância determinada, possibilitando o cálculo das concentrações finais por meio da curva de calibração. 


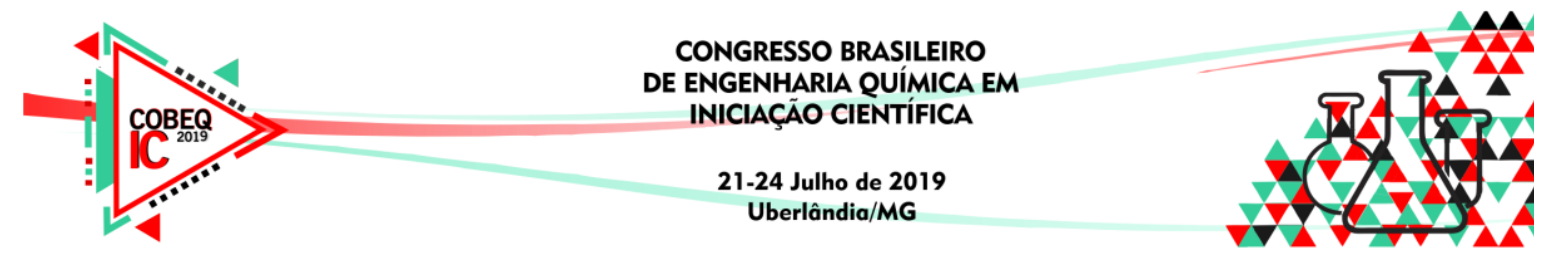

\section{RESULTADOS E DISCUSSÃO}

\subsection{Conversão}

Gravimetria: A Figura 2 apresenta a evolução da conversão, calculada considerando-se a razão entre a massa seca e a massa retirada do reator e as frações mássicas de monômero em cada instante. Para a reação de formação da casca, considerou-se apenas os monômeros dispersos no meio reacional após a adição de DVB, de modo que, a partir de 90 min, a conversão voltasse a ser nula. Tal como esperado, a curva tende a estabilidade nos instantes finais de reação. Na primeira parte do ensaio, a conversão final de monômeros foi de $65 \%$ e, na segunda parte, de aproximadamente $30 \%$. Considerando-se o estireno como reagente limitante na primeira reação, conclui-se que a conversão máxima do núcleo é da ordem de $67 \%$, o que demonstra a adequação do resultado. Já a conversão da casca ficou aquém do esperado, próximo a 100\% (Chen et al, 2016), devido à insuficiência da agitação para garantir um perfil homogêneo de temperaturas dentro do reator e às altas quantidades de inibidores no agente reticulante, para conservação do produto.

Figura 2 - Conversão dos monômeros na etapa de síntese, medida por gravimetria.

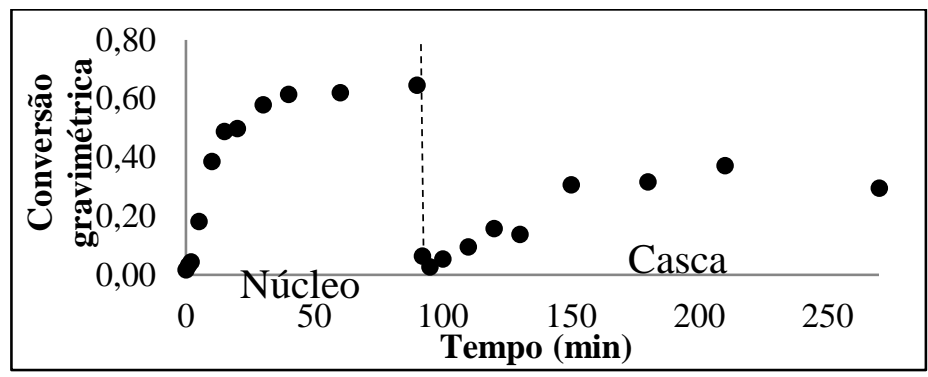

\subsection{Diâmetro das Partículas}

Espalhamento Dinâmico de Luz (EDL): O diâmetro médio, determinado por meio de EDL, demonstrou valores acima de $1 \mu \mathrm{m}$, indicando que a técnica, apesar de apresentar baixo custo e pouca complexidade de execução, pode não ser a mais adequada para este sistema. De acordo com Chen et al. (2016), a primeira etapa da reação deveria gerar núcleos monodispersos por volta de $580 \mathrm{~nm}$ e, a segunda, partículas com casca e cerca de $760 \mathrm{~nm}$. Com o avanço da conversão de reagentes, ocorre o crescimento de diâmetro do núcleo e da casca, o que pode ser observado na Figura 3.

Figura 3 - Diâmetro médio das partículas.

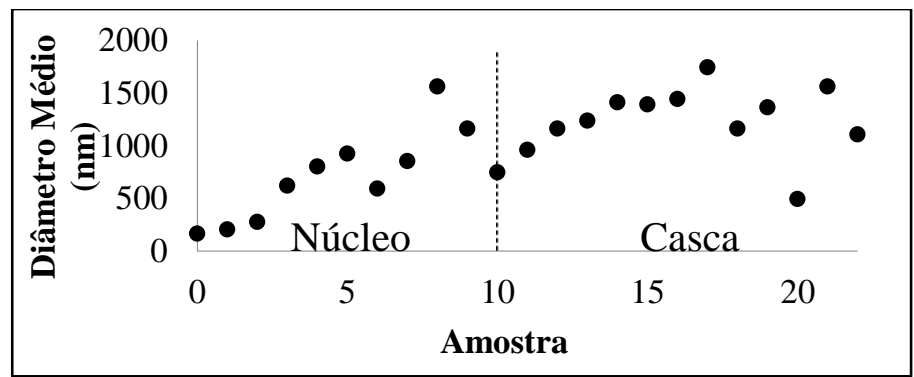




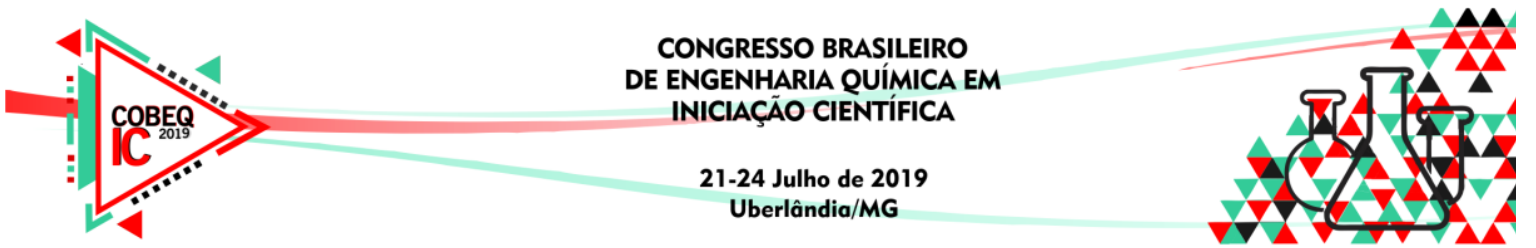

Microscopia eletrônica de varredura (MEV): As imagens obtidas por MEV (Figura 4) permitem analisar as mudanças nos aspectos morfológicos após os processos de centrifugação, lavagem, secagem e funcionalização. Observa-se que, após todas as etapas, as formas esféricas das partículas se mantêm estáveis, devido, principalmente, ao invólucro altamente reticulado que possui excelente resistência mecânica. Tal consideração é importante, uma vez que, geralmente, um adsorvente eficaz deve ser um material sólido com alta estabilidade mecânica e química, o que o torna adequado para aplicações sob condições severas, podendo ser facilmente separado e reutilizado posteriormente.

Figura 4 - MEV após os processos de (a) reação, (b) centrifugação e (c) funcionalização.

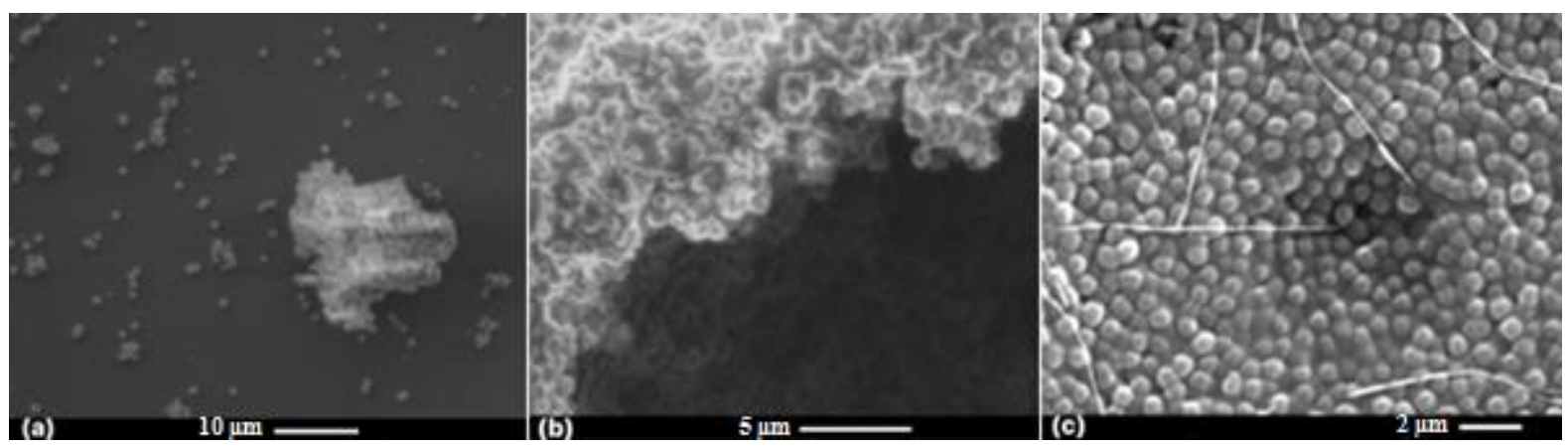

Os diâmetros observados nas microscopias diferem dos obtidos por EDL, porém as análises por MEV apontam para a faixa entre 600 e $700 \mathrm{~nm}$. A aglomeração das partículas na Figura 4 (a) pode ser justificada por efeitos de coalescência ou coagulação, normalmente decorrentes de concentrações altas de monômeros e baixas de iniciadores. Com a limitação dos demais componentes para formação da partícula final e a ausência de cargas suficientes no meio para manter as partículas suspensas, ocorre a desestabilização do sistema, levando à aglomeração do excesso de monômeros, como exposto por Oropeza (2011). Com o passar do tempo, considerando-se também o armazenamento das amostras, isso pode levar à formação das estruturas retratadas na imagem.

\subsection{Testes de Adsorção}

Os dados experimentais da curva de equilíbrio de adsorção foram ajustados por uma isoterma de Langmuir, onde $C_{e}(M)$ é a concentração de corante no equilíbrio; $Q_{e}(\mathrm{mg} / \mathrm{g})$ é a capacidade de adsorção do polímero; $\mathrm{Q}_{\max }(\mathrm{mg} / \mathrm{g})$ é a capacidade máxima de adsorção; e $\mathrm{K}_{\mathrm{L}}$ $(\mathrm{L} / \mathrm{mg})$ é o parâmetro de afinidade. Este modelo, dado na Equação 1, considera a hipótese de adsorção homogênea e em monocamada na superfície adsorvente, a qual contém um número finito de sítios de ligação. Outros modelos, como Freundlich, Redlich-Peterson e KobleCorrigan serão abordados em trabalhos futuros. A Figura 5 apresenta os resultados destas análises, bem como observações qualitativas dos testes.

$$
\frac{C_{e}}{Q_{e}}=\frac{C_{e}}{Q_{\max }}+\frac{1}{Q_{\max } K_{L}}
$$




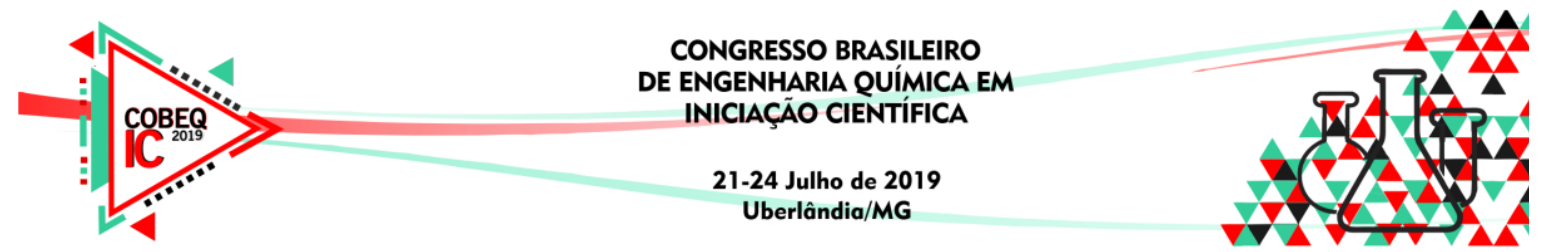

Figura 5 - Soluções de azul de metileno (a) antes e (b) depois da adsorção e isotermas de (c) equilíbrio, com dados experimentais e teóricos e (d) Langmuir.

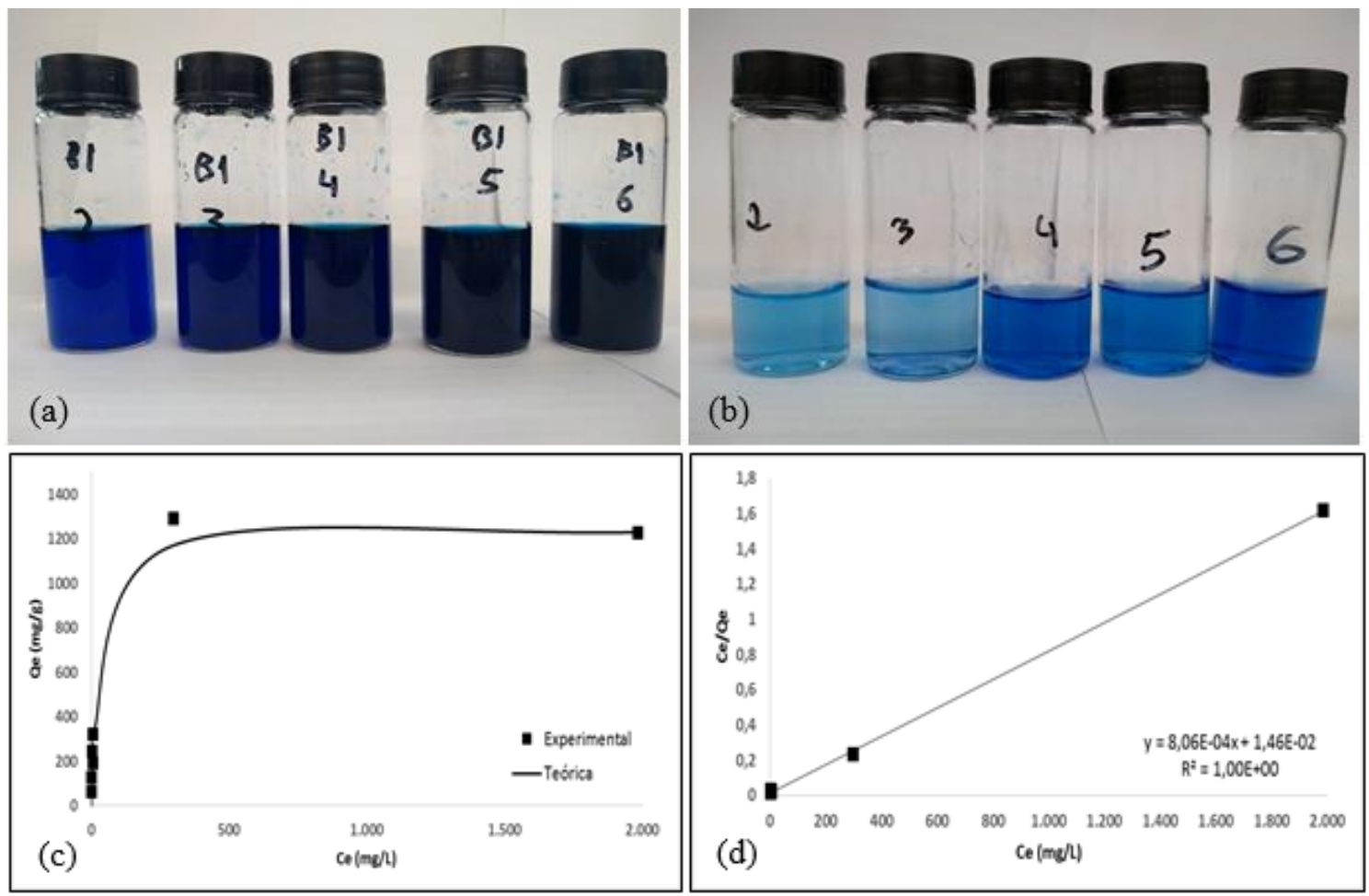

São visualmente notáveis a homogeneidade e o clareamento entre as soluções 5 (a) e (b). A adsorção de azul de metileno ao copolímero se dá, principalmente, devido à atração eletrostática entre os carboxilatos do polímero, carregados negativamente, e os grupos amônio no corante catiônico, carregados positivamente. O fenômeno ocorre de forma espontânea, levando a uma eficiente remoção de corante sob baixas concentrações no meio. Isso ocorre pois os grupos anidrido na superfície polimérica são convertidos em carboxilatos após a hidrólise e a estrutura reticulada encontra-se ligeiramente inchada em meio aquoso, permitindo que os íons carboxilato conjugem-se com as moléculas de corante. A estrutura oca fornece uma alta área de contato, enquanto os mesoporos interconectados no invólucro servem como via de difusão das moléculas de corante no interior do polímero. Com o aumento da concentração no meio, os sítios de ligação na superfície tornam-se saturados de moléculas, reduzindo a disponibilidade de sítios ativos vagos na superfície porosa. Então, as moléculas de corante devem penetrar na camada superficial e, em seguida, se difundir dentro da casca reticulada; assim, a taxa de adsorção diminui drasticamente e a quantidade de corante adsorvido passa a aumentar lentamente até atingir o valor aproximadamente constante da adsorção de equilíbrio. Além disso, este comportamento também é ocasionado pela formação de um campo eletrostático em torno da superfície do adsorvente, promovendo a repulsão eletrostática dos compostos no meio. Deste modo, dispensam-se maior quantidade de medições para concentrações superiores àquela de equilíbrio. O fenômeno é exemplificado na Figura 5 (c), onde podem ser observados os pontos experimentais e a curva teórica calculada a partir do modelo de Langmuir.

O ajuste fornecido pelo modelo de Langmuir em 5 (d) apresenta boa linearidade, com alto coeficiente de correlação, o que sugere a validade das hipóteses subentendidas pelo modelo. 


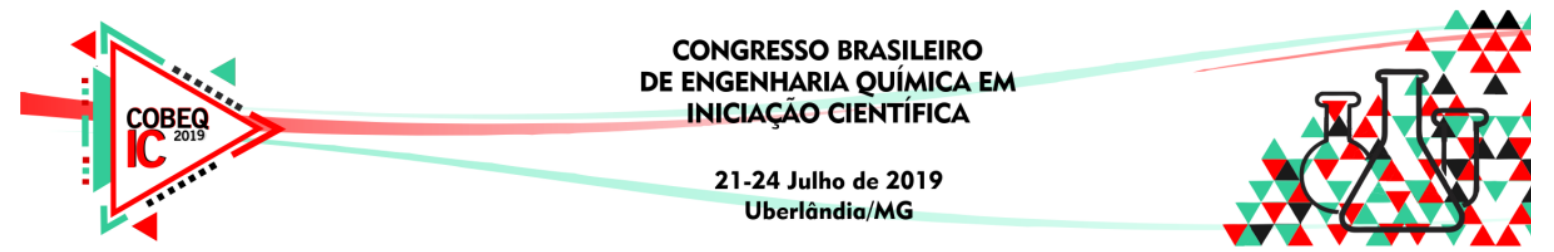

O parâmetro $Q_{\max }$ foi calculado em 1240,69 mg/g, o que corresponde a uma elevação de $0,71 \%$ em relação ao proposto por Chen et al. (2016) (1232 mg/g); já o parâmetro de afinidade $\mathrm{K}_{\mathrm{L}}$, apresentou valor de $0,0552 \mathrm{l} / \mathrm{mg}$, inferior ao esperado. Os desvios apresentados podem estar intimamente relacionados à dificuldade no controle do $\mathrm{pH}$ do meio; é sabido que o aumento dos valores de $\mathrm{pH}$ leva ao crescimento da capacidade adsortiva do copolímero, devido à maior presença de íons -COO- nestas condições.

\section{CONCLUSÃO}

A síntese das micropartículas de poli (estireno-co-anidrido maleico) formados in situ como template, segundo o modelo proposto por Chen et al. (2016), demonstraram elevada conversão, de $65 \%$, em oposição ao valor de $30 \%$ observado na formação da casca. Na análise por MEV, as partículas analisadas apresentaram formas esféricas com diâmetros entre $600 \mathrm{e}$ $700 \mathrm{~nm}$, estáveis durante as etapas de reação, centrifugação, lavagem, secagem e funcionalização, demonstrando a qualidade das resistências química e mecânica do invólucro reticulado. Estas características comprovam a viabilidade de uso do adsorvente sob condições variadas, dada sua possibilidade de recuperação.

Com o alto teor de anidridos funcionalizados com grupos carboxilato em excesso no meio reacional com $\mathrm{pH} 7$, tais grupos mostram-se carregados negativamente, de forma que se mostram eficientes para adsorção de corantes de carga positiva, como é o caso do azul de metileno. Em relação aos testes de adsorção realizados com este corante, observou-se uma capacidade máxima de adsorção de 1240,69 mg/g, valor de acordo com Chen et al. (2016).

Por fim, espera-se que, devido à estrutura multicamadas e à alta densidade de grupos funcionais, as micropartículas porosas sintetizadas possam apresentar potencial aplicabilidade na recuperação de soluções contaminadas com corantes, adsorção de íons de metais pesados, suportes de catalisadores, entre outras aplicações.

\section{REFERENCIAS}

CHEN, D.; WANG, L.; MA, Y.; YANG, W. Super-adsorbent material based on functional polymer particles with a multilevel porous structure. NPG Asia Materials, 8 (301), pp. 1-9, 2016.

OROPEZA, M. V. C. Síntese e caracterização de nanopartículas núcleo-casca de poliestireno e polimetacrilato de metila obtidas por polimerização em emulsão sem emulsificante e fotoiniciada. Tese (Doutorado em Engenharia Química) - EPUSP, 2011.

QIN, Y.; WANG, L.; ZHAO, C.; CHEN, D.; MA, Y; YANG, W. Ammonium-Functionalized Hollow Polymer Particles As a pH-Responsive Adsorbent for Selective Removal of Acid Dye. ACS Appl. Mater. Interfaces 8, 16690-16698, 2016.

SANTOS, S. C.; BOAVENTURA, R. A. Adsorption modelling of textile dyes by sepiolite. Appl. Clay Sci. 42, 137-145, 2008. 